\title{
Randomization Method
}

National Cancer Institute

\section{Source}

National Cancer Institute. Randomization Method. NCI Thesaurus. Code C147140.

The type of randomization method used to assign subjects to treatment groups or cohorts. 